\title{
Нарушение локальной электронейтральности в квантовой яме полупроводникового лазера с асимметричными барьерными слоями
}

\author{
() Л.В. Асрян ${ }^{1}$, Ф.И. Зубов ${ }^{2}$ Ю.С. Балезина (Полубавкина) ${ }^{2}$, Э.И. Моисеев $^{2}$, М.Е. Муретова ${ }^{2}$, \\ Н.В. Крыжановская ${ }^{2}$, М.В. Максимов ${ }^{2}$, А.Е. Жуков ${ }^{2}$ \\ ${ }^{1}$ Virginia Polytechnic Institute and State University, \\ Blacksburg, Virginia 24061, USA \\ ${ }^{2}$ Санкт-Петербургский национальный исследовательский Академический университет \\ Российской академии наук, \\ 194021 Санкт-Петербург, Россия \\ E-mail: asryan@vt.edu
}

(Получена 28 марта 2018 г. Принята к печати 4 апреля 2018 г.)

Разработана самосогласованная модель для расчета пороговых и мощностных характеристик полупроводниковых лазеров на квантовой яме с асимметричными барьерными слоями. Модель, основанная на системе скоростных уравнений, использует универсальное условие глобальной зарядовой нейтральности в лазерной структуре. Рассчитаны концентрации электронов и дырок в волноводной области и в квантовой яме и концентрация фотонов стимулированного излучения. Показано, что локальная нейтральность в квантовой яме сильно нарушена, особенно при высоких токах инжекции. Нарушение нейтральности в квантовой яме приводит к зависимости концентраций электронов и дырок в ней от тока инжекции в режиме лазерной генерации - в рассмотренной нами структуре концентрация электронов в квантовой яме уменьшается, а концентрация дырок увеличивается с ростом тока инжекции. В условиях идеального функционирования асимметричных барьерных слоев, когда имеет место полное подавление электроннодырочной рекомбинации в волноводной области, нарушение нейтральности в квантовой яме практически не сказывается на зависимости мощности выходного оптического излучения от тока инжекции - квантовая эффективность близка к единице, а ватт-амперная характеристика линейна. Нарушение нейтральности в квантовой яме приводит, тем не менее, к ослаблению температурной зависимости порогового тока и, таким образом, повышению характеристической температуры $T_{0}$ лазера.

DOI: 10.21883/FTP.2018.12.46768.8876

\section{1. Введение}

Использование асимметричных барьерных слоев (АБС, asymmetric barrier layers - ABLs) [1-4], наряду с двойной туннельной инжекцией носителей заряда [5-8], было предложено ранее для подавления паразитной электронно-дырочной рекомбинации в лазерном волноводе и тем самым коренного улучшения приборных характеристик лазеров с низкоразмерной активной областью. В этих обоих типах лазеров подавление рекомбинации достигается посредством предотвращения одновременного (биполярного) присутствия электронов и дырок где-либо в структуре за исключением активной области. В лазерах с АБС (рис. 1) для блокирования биполярной заселенности в волноводной области (области оптического ограничения, optical confinement layer OCL) используются два асимметричных барьерных слоя. Один из этих слоев (левый на рис. 1) не препятствует попаданию электронов, инжектированных из $n$-эмиттера, в активную область, но препятствует утечке дырок из активной области в левую часть волноводной области, в которой имеется значительная концентрация электронов. Другой АБС (правый на рис. 1) позволяет дыркам, инжектированным из $p$-эмиттера, беспрепятственно достигнуть активной области, но блокирует электронную утечку из этой области в правую часть волноводной области, где имеются в изобилии дырки.
В работе [9] были рассчитаны рабочие характеристики лазеров с АБС с активной областью в виде квантовой ямы (КЯ). При расчетах в работе [9] учитывалась асимметрия между заполнением электронных и дырочных состояний, однако в то же время предполагалась локальная электронейтральность в КЯ, т.е. равенство друг другу двумерных концентраций электронов и дырок в КЯ. Однако, как было показано ранее [10-13], ввиду асимметрии между электронными и дырочными параметрами, даже в обычных лазерных структурах, не содержащих АБС, имеет место нарушение локальной электронейтральности в активной области. В лазерах с АБС, в дополнение к асимметрии между электронными и дырочными параметрами, имеет место асим-

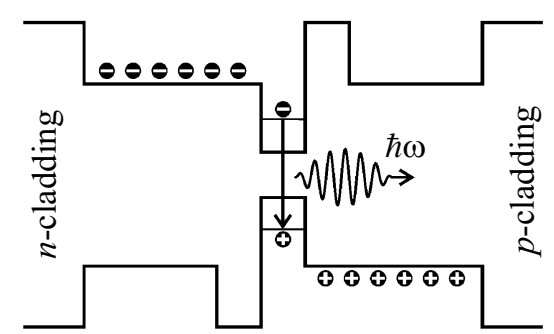

Рис. 1. Схематическая зонная энергетическая диаграмма лазера с асимметричными барьерными слоями. 
метричное пространственное распределение носителей противоположного знака в волноводной области (на рис. 1 электроны присутствуют только в ее левой части, дырки - в правой) и, таким образом, нарушение локальной электронейтральности в активной области может проявляться еще сильнее. В данной работе представлена самосогласованная модель для расчета пороговых и мощностных характеристик полупроводниковых лазеров на КЯ с АБС, учитывающая возможное нарушение электронейтральности в активной области и использующая универсальное условие глобальной зарядовой нейтральности в структуре.

\section{2. Основные уравнения}

Наша модель основана на следующей системе скоростных уравнений:

$$
\begin{aligned}
& b_{1} \frac{\partial n_{\mathrm{L}}^{\mathrm{OCL}}}{\partial t}=\frac{j}{e}+\frac{n^{\mathrm{QW}}}{\tau_{n, \mathrm{esc}}}-v_{n, \text { capt }, 0}\left(1-f_{n}\right) n_{\mathrm{L}}^{\mathrm{OCL}} \text {, } \\
& b_{2} \frac{\partial p_{\mathrm{R}}^{\mathrm{OCL}}}{\partial t}=\frac{j}{e}+\frac{p^{\mathrm{QW}}}{\tau_{p, \text { esc }}}-v_{p, \text { capt }, 0}\left(1-f_{p}\right) p_{\mathrm{R}}^{\mathrm{OCL}}, \\
& \frac{\partial n^{\mathrm{QW}}}{\partial t}=v_{n, \text { capt }, 0}\left(1-f_{n}\right) n_{\mathrm{L}}^{\mathrm{OCL}}-\frac{n^{\mathrm{QW}}}{\tau_{n, \mathrm{esc}}}-B_{2 \mathrm{D}} n^{\mathrm{QW}} p^{\mathrm{QW}} \\
& -c_{g} g^{\max }\left(f_{n}+f_{p}-1\right) n_{\mathrm{ph}}, \\
& \frac{\partial p^{\mathrm{QW}}}{\partial t}=v_{p, \text { capt }, 0}\left(1-f_{p}\right) p_{\mathrm{R}}^{\mathrm{OCL}}-\frac{p^{\mathrm{QW}}}{\tau_{p, \text { esc }}}-B_{2 \mathrm{D}} n^{\mathrm{QW}} p^{\mathrm{QW}} \\
& -c_{g} g^{\max }\left(f_{n}+f_{p}-1\right) n_{\mathrm{ph}}, \\
& \frac{\partial n_{\mathrm{ph}}}{\partial t}=c_{g} g^{\max }\left(f_{n}+f_{p}-1\right) n_{\mathrm{ph}}-c_{g} \beta n_{\mathrm{ph}},
\end{aligned}
$$

в которой неизвестными величинами являются трехмерная (3D) концентрация $n_{\mathrm{L}}^{\mathrm{OCL}}$ свободных электронов в левой части волноводной области, трехмерная концентрация $p_{\mathrm{R}}^{\mathrm{OCL}}$ свободных дырок в правой части волноводной области, двумерные (2D) концентрации $n^{\mathrm{QW}}$ и $p^{\mathrm{QW}}$ электронов и дырок, локализованных в КЯ, и концентрация (на единицу площади контакта) фотонов стимулированного излучения $n_{\mathrm{ph}}$.

В уравнения $(1)-(5)$ также входят величины $f_{n}$ и $f_{p}$, представляющие собой степени заполнения (заселенности) состояний, соответствующих нижнему краю электронной и верхнему краю дырочной подзон размерного квантования в КЯ. Однако эти величины не являются независимыми - они выражаются через концентрации электронов и дырок в КЯ [14-16],

$$
f_{n}=1-\exp \left(-\frac{n^{\mathrm{QW}}}{N_{c}^{2 \mathrm{D}}}\right), \quad f_{p}=1-\exp \left(-\frac{p^{\mathrm{QW}}}{N_{v}^{2 \mathrm{D}}}\right),
$$

где $N_{c, v}^{2 \mathrm{D}}=m_{e, h h}^{\mathrm{QW}} T /\left(\pi \hbar^{2}\right)-$ двумерные эффективные плотности состояний в зоне проводимости и валентной зоне в КЯ, $m_{e, h h}^{\mathrm{QW}}-$ эффективные массы электронов и дырок в КЯ, $T$ - температура в энергетических единицах.

Параметрами в уравнениях (1)-(5) являются следующие величины: $b_{1}, b_{2}$ - толщины левой и правой частей волноводной области (как правило, они равны), jплотность тока инжекции, $e-$ заряд электрона, $\tau_{n \text {, esc }}$ и $\tau_{p \text {, еsс }}$ - времена термических выбросов электронов и дырок из КЯ в волноводную область; $v_{n, \text { capt, } 0}$ и $v_{p, \text { capt }, 0}$ - скорости захвата электронов и дырок из волноводной области в пустую (при $f_{n}=f_{p}=0$ ) КЯ, измеряемые в см/c; $B_{2 \mathrm{D}}$ - коэффициент спонтанной излучательной рекомбинации в двумерной области (КЯ), измеряемый в см ${ }^{2} / \mathrm{c}[17] ; c_{g}-$ групповая скорость света; $g^{\max }-$ максимальный модовый коэффициент усиления в КЯ; $\beta=[1 /(2 L)] \ln \left[1 /\left(R_{1} R_{2}\right)\right]$ - потери, связанные с выходом излучения из резонатора, $L-$ длина лазерного резонатора Фабри-Перо; $R_{1}, R_{2}$ - коэффициенты отражения торцевых зеркал резонатора.

Времена термических выбросов из КЯ в волноводную область могут быть выражены через скорости захвата из волноводной области в пустую КЯ $[8,18]$. В случае нелегированных волноводной области и КЯ эти выражения имеют следующий вид $[8,18]$ :

$$
\begin{aligned}
\tau_{n, \mathrm{esc}} & =\frac{1}{v_{n, \text { capt }, 0}\left(1-f_{n}\right)} \frac{N_{c}^{2 \mathrm{D}}}{n_{1}}, \\
\tau_{p, \text { esc }} & =\frac{1}{v_{p, \text { capt }, 0}\left(1-f_{p}\right)} \frac{N_{v}^{2 \mathrm{D}}}{p_{1}},
\end{aligned}
$$

где

$$
\begin{aligned}
& n_{1}=N_{c}^{3 \mathrm{D}} \exp \left(-\frac{\Delta E_{c}-\varepsilon_{n}^{\mathrm{QW}}}{T}\right), \\
& p_{1}=N_{v}^{3 \mathrm{D}} \exp \left(-\frac{\Delta E_{v}-\varepsilon_{p}^{\mathrm{QW}}}{T}\right),
\end{aligned}
$$

где $N_{c, v}^{3 \mathrm{D}}=2\left[m_{c, v}^{\mathrm{OCL}} T /\left(2 \pi \hbar^{2}\right)\right]^{3 / 2}$ - трехмерные эффективные плотности состояний в зоне проводимости и валентной зоне в волноводной области, $m_{c, v}^{\mathrm{OCL}}-$ эффективные массы электронов и дырок в волноводной области, $\Delta E_{c, v}$ - разрывы краев зоны проводимости и валентной зоны на гетероконтакте КЯ и волноводной области, $\varepsilon_{n}^{\mathrm{QW}}$ и $\varepsilon_{p}^{\mathrm{QW}}-$ энергии соответственно нижнего и верхнего краев электронной и дырочной подзон размерного квантования в КЯ.

Как отражено в уравнениях (1)-(5), в этой статье предполагается, что оба АБС функционируют идеально и, следовательно, нет свободных электронов (дырок) в правой (левой) части волноводной области, т. е. $n_{\mathrm{R}}^{\mathrm{OCL}}=0$ и $p_{\mathrm{L}}^{\mathrm{OCL}}=0$, и, таким образом, рекомбинация в волноводной области полностью подавлена.

Уравнения (1)-(5) представляют собой систему для нахождения пяти неизвестных величин (четырех концентраций носителей $n_{\mathrm{L}}^{\mathrm{OCL}}, p_{\mathrm{R}}^{\mathrm{OCL}}, n^{\mathrm{QW}}, p^{\mathrm{QW}}$ и концентрации фотонов $\left.n_{\mathrm{ph}}\right)$. Далее показано, что четыре из пяти неизвестных в этой системе можно выразить через пятую (для определеннности это будет $n^{\mathrm{QW}}$ ) и далее свести 
решение системы к решению алгебраического уравнения для нахождения этой неизвестной $\left(n^{\mathrm{QW}}\right)$.

Складывая (1) и (3), мы получаем

$$
\begin{aligned}
\frac{\partial}{\partial t}\left(b_{1} n_{\mathrm{L}}^{\mathrm{OCL}}+n^{\mathrm{QW}}\right)= & \frac{j}{e}-B_{2 \mathrm{D}} n^{\mathrm{QW}} p^{\mathrm{QW}} \\
& -c_{g} g^{\max }\left(f_{n}+f_{p}-1\right) n_{\mathrm{ph}} .
\end{aligned}
$$

Складывая (2) и (4), получаем

$$
\begin{aligned}
\frac{\partial}{\partial t}\left(b_{2} p_{\mathrm{R}}^{\mathrm{OCL}}+p^{\mathrm{QW}}\right)= & \frac{j}{e}-B_{2 \mathrm{D}} n^{\mathrm{QW}} p^{\mathrm{QW}} \\
& -c_{g} g^{\max }\left(f_{n}+f_{p}-1\right) n_{\mathrm{ph}} .
\end{aligned}
$$

Сравнение (9) и (10) дает

$$
\frac{\partial}{\partial t}\left[e\left(b_{2} p_{\mathrm{R}}^{\mathrm{OCL}}+p^{\mathrm{QW}}\right)-e\left(b_{1} n_{\mathrm{L}}^{\mathrm{OCL}}+n^{\mathrm{QW}}\right)\right]=0 .
$$

Выражение в квадратных скобках в (11) представляет собой полный заряд на единицу площади контакта в лазерной структуре. Уравнение (11), таким образом, представляет собой условие сохранения полного заряда в структуре. Так как лазерная структура изначально не заряжена, мы получаем из (11) универсальное условие ее глобальной электронейтральности в следующем виде:

$$
b_{1} n_{\mathrm{L}}^{\mathrm{OCL}}+n^{\mathrm{QW}}=b_{2} p_{\mathrm{R}}^{\mathrm{OCL}}+p^{\mathrm{QW}},
$$

представляющее собой равенство суммарного заряда (на единицу площади) электронов в КЯ и левой части волноводной области суммарному заряду дырок в КЯ и правой части волноводной области.

Мы рассматриваем непрерывный режим работы лазера и, таким образом, будем решать скоростные уравнения (1)-(5) для стационарного случая $(\partial / \partial t=0$ во всех уравнениях).

Из (1) и (2) имеем для $n_{\mathrm{L}}^{\mathrm{OCL}}$ и $p_{\mathrm{R}}^{\mathrm{OCL}}$

$$
\begin{aligned}
n_{\mathrm{L}}^{\mathrm{OCL}} & =\frac{j}{e v_{n, \mathrm{capt}, 0}\left(1-f_{n}\right)}+\frac{1}{\tau_{n, \mathrm{esc}}} \frac{1}{v_{n, \mathrm{capt}, 0}\left(1-f_{n}\right)} n^{\mathrm{QW}}, \\
p_{\mathrm{R}}^{\mathrm{OCL}} & =\frac{j}{e v_{p, \text { capt }, 0}\left(1-f_{p}\right)}+\frac{1}{\tau_{p, \text { esc }}} \frac{1}{v_{p, \text { capt }, 0}\left(1-f_{p}\right)} p^{\mathrm{QW}} .
\end{aligned}
$$

С учетом (7) уравнения (13) и (14) можно записать в следующем виде:

$$
\begin{aligned}
n_{\mathrm{L}}^{\mathrm{OCL}} & =\frac{j}{e v_{n, \text { capt }, 0}\left(1-f_{n}\right)}+n_{1} \frac{n^{\mathrm{QW}}}{N_{c}^{2 \mathrm{D}}}, \\
p_{\mathrm{R}}^{\mathrm{OCL}} & =\frac{j}{e v_{p, \text { capt }, 0}\left(1-f_{p}\right)}+p_{1} \frac{p^{\mathrm{QW}}}{N_{v}^{2 \mathrm{D}}} .
\end{aligned}
$$

Используя (6) в уравнении (15), мы окончательно выражаем $n_{\mathrm{L}}^{\mathrm{OCL}}$ через $n^{\mathrm{QW}}$ :

$$
n_{\mathrm{L}}^{\mathrm{OCL}}=\frac{j}{e v_{n, \text { capt }, 0}} \exp \left(\frac{n^{\mathrm{QW}}}{N_{c}^{2 \mathrm{D}}}\right)+n_{1} \frac{n^{\mathrm{QW}}}{N_{c}^{2 \mathrm{D}}} .
$$

Уравнение (5) в стационарном режиме дает

$$
f_{n}+f_{p}-1=\frac{\beta}{g^{\max }}
$$

что представляет собой условие лазерной генерации. С помощью (6) уравнение (18) может быть записано в виде

$$
1-\exp \left(-\frac{n^{\mathrm{QW}}}{N_{c}^{2 \mathrm{D}}}\right)-\exp \left(-\frac{p^{\mathrm{QW}}}{N_{v}^{2 \mathrm{D}}}\right)=\frac{\beta}{g^{\max }} .
$$

Из (19) можно выразить $p^{\mathrm{QW}}$ через $n^{\mathrm{QW}}$ следующим образом:

$$
p^{\mathrm{QW}}=N_{v}^{2 \mathrm{D}} \ln \frac{1}{1-\frac{\beta}{g^{\mathrm{max}}}-\exp \left(-\frac{n^{\mathrm{QW}}}{N_{c}^{2 \mathrm{D}}}\right)} .
$$

Из (18) для $\left(1-f_{p}\right)$ имеем

$$
1-f_{p}=f_{n}-\frac{\beta}{g^{\max }}
$$

или, используя (6),

$$
1-f_{p}=1-\frac{\beta}{g^{\max }}-\exp \left(-\frac{n^{\mathrm{QW}}}{N_{c}^{2 \mathrm{D}}}\right) .
$$

Используя (20) и (22) в уравнении (16), мы окончательно выражаем $p_{\mathrm{R}}^{\mathrm{OCL}}$ через $n^{\mathrm{QW}}$,

$$
\begin{aligned}
p_{\mathrm{R}}^{\mathrm{OCL}}= & \frac{j}{e v_{p, \text { capt }, 0}\left[1-\frac{\beta}{g^{\max }}-\exp \left(-\frac{n^{\mathrm{QW}}}{N_{c}^{2 \mathrm{D}}}\right)\right]} \\
& +p_{1} \ln \frac{1}{1-\frac{\beta}{g^{\max }}-\exp \left(-\frac{n^{\mathrm{QW}}}{N_{c}^{2 \mathrm{D}}}\right)} .
\end{aligned}
$$

Подставив выражения (17), (20) и (23) для $n_{\mathrm{L}}^{\mathrm{OCL}}$, $p^{\mathrm{QW}}$ и $p_{\mathrm{R}}^{\mathrm{OCL}}$ в условие глобальной электронейтральности (12), мы получаем следующее трансцендентное алгебраическое уравнение для нахождения $n$ QW:

$$
\begin{aligned}
b_{1}\left[\frac{j}{e v_{n, \text { capt }, 0}}\right. & \left.\exp \left(\frac{n^{\mathrm{QW}}}{N_{c}^{2 \mathrm{D}}}\right)+n_{1} \frac{n^{\mathrm{QW}}}{N_{c}^{2 \mathrm{D}}}\right]+n^{\mathrm{QW}} \\
= & b_{2}\left\{\frac{j}{e v_{p, \text { capt }, 0}\left[1-\frac{\beta}{g^{\max }}-\exp \left(-\frac{n^{\mathrm{QW}}}{N_{c}^{2 \mathrm{D}}}\right)\right]}\right. \\
& \left.+p_{1} \ln \frac{1}{1-\frac{\beta}{g^{\max }}-\exp \left(-\frac{n^{\mathrm{QW}}}{N_{c}^{2 \mathrm{D}}}\right)}\right\} \\
& +N_{v}^{2 \mathrm{D}} \ln \frac{1}{1-\frac{\beta}{g^{\max }}-\exp \left(-\frac{n^{\mathrm{QW}}}{N_{c}^{2 \mathrm{D}}}\right)} .
\end{aligned}
$$

Решение уравнения (24) дает нам двумерную концентрацию $n^{\mathrm{QW}}$ электронов в КЯ в виде функции от плотности тока инжекции $j$ и параметров лазерной 
структуры. Имея $n^{\mathrm{QW}}$, мы далее вычисляем $n_{\mathrm{L}}^{\mathrm{OCL}}, p^{\mathrm{QW}}$ и $p_{\mathrm{R}}^{\mathrm{OCL}}$ с помощью уравнений $(17),(20)$ и (23).

Концентрация фотонов в резонаторе также выражается через $n^{\mathrm{QW}}$. Покажем это: из уравнений (9) и (10) в стационарном режиме мы имеем для плотности тока инжекции

$$
j=e B_{2 \mathrm{D}} n^{\mathrm{QW}} p^{\mathrm{QW}}+e c_{g} g^{\max }\left(f_{n}+f_{p}-1\right) n_{\mathrm{ph}} .
$$

Используя (18) и введя время жизни фотонов в резонаторе

$$
\tau_{\mathrm{ph}}=\frac{1}{c_{g} \beta},
$$

для плотности тока инжекции имеем

$$
j=e B_{2 \mathrm{D}} n^{\mathrm{QW}} p^{\mathrm{QW}}+e \frac{n_{\mathrm{ph}}}{\tau_{\mathrm{ph}}} .
$$

Из (27) для концентрации фотонов стимулированного излучения имеем

$$
n_{\mathrm{ph}}=\tau_{\mathrm{ph}}\left(\frac{j}{e}-B_{2 \mathrm{D}} n^{\mathrm{QW}} p^{\mathrm{QW}}\right)
$$

где $p^{\mathrm{QW}}$ выражается через $n^{\mathrm{QW}}$ согласно (20).

Решение уравнения (24) позволяет нам далее вычислить ватт-амперную характеристику (ВтАХ) лазера, т.е. мощность выходного оптического излучения в зависимости от плотности тока инжекции $[19,20]$ :

$$
\begin{aligned}
P(j) & =\frac{\hbar \omega}{e} S j_{\mathrm{stim}}(j)=\hbar \omega \frac{n_{\mathrm{ph}}}{\tau_{\mathrm{ph}}} S \\
& =\frac{\hbar \omega}{e} S\left[j-e B_{2 \mathrm{D}} n^{\mathrm{QW}}(j) p^{\mathrm{QW}}(j)\right] \\
& =\frac{\hbar \omega}{e} S\left(j-j_{\mathrm{tn}}\right) \eta_{\mathrm{int}}(j),
\end{aligned}
$$

где $\hbar \omega-$ энергия фотона, $S=W L-$ площадь полоскового контакта, $W-$ ширина контакта, $j_{\text {th }}-$ плотность порогового тока.

Плотность тока стимулированной рекомбинации и внутренняя дифференциальная квантовая эффективность (эффективность стимулированной излучательной рекомбинации), входящие в (29), определяются следующим образом [21]:

$$
\begin{aligned}
j_{\mathrm{stim}}=e \frac{n_{\mathrm{ph}}}{\tau_{\mathrm{ph}}} & =j-e B_{2 \mathrm{D}} n^{\mathrm{QW}} p^{\mathrm{QW}}, \\
\eta_{\text {int }} & =\frac{j_{\mathrm{stim}}}{j-j_{\mathrm{th}}} .
\end{aligned}
$$

Плотность порогового тока $j_{\text {th }}$ находится как корень уравнения

$$
n_{\mathrm{ph}}(j)=0
$$

или, используя (28),

$$
j-e B_{2 \mathrm{D}} n^{\mathrm{QW}}(j) p^{\mathrm{QW}}(j)=0 .
$$

Уравнение (31) может быть записано в виде

$$
\begin{aligned}
\eta_{\text {int }}(j) & =\frac{j-e B_{2 \mathrm{D}} n^{\mathrm{QW}}(j) p^{\mathrm{QW}}(j)}{j-e B_{2 \mathrm{D}} n^{\mathrm{QW}}\left(j_{\text {th }}\right) p^{\mathrm{QW}}\left(j_{\text {th }}\right)} \\
& =\frac{j-j_{\text {spon }}^{\mathrm{QW}}(j)}{j-j_{\text {spon, th }}^{\mathrm{QW}}}=\frac{j-j_{\text {spon }}^{\mathrm{QW}}(j)}{j-j_{\text {th }}}
\end{aligned}
$$

где мы ввели плотность тока спонтанной излучательной рекомбинации в КЯ

$$
j_{\text {spon }}^{\mathrm{QW}}(j)=e B_{2 \mathrm{D}} n^{\mathrm{QW}}(j) p^{\mathrm{QW}}(j)
$$

и представили плотность порогового тока в виде

$$
j_{\text {th }}=j_{\text {spon, th }}^{\mathrm{QW}}=e B_{2 \mathrm{D}} n^{\mathrm{QW}}\left(j_{\mathrm{th}}\right) p^{\mathrm{QW}}\left(j_{\text {th }}\right)=e B_{2 \mathrm{D}} n_{\mathrm{th}}^{\mathrm{QW}} p_{\mathrm{th}}^{\mathrm{QW}},
$$

где

$$
n_{\mathrm{th}}^{\mathrm{QW}}=n^{\mathrm{QW}}\left(j_{\text {th }}\right), \quad p_{\mathrm{th}}^{\mathrm{QW}}=p^{\mathrm{QW}}\left(j_{\text {th }}\right)
$$

являются пороговыми значениями концентраций электронов и дырок в КЯ.

\section{3. Обсуждение результатов}

В этом разделе приводятся результаты расчетов, произведенных с помощью нашей модели для лазерных структур, излучающих на длинах волн $\lambda_{0}=808 \mathrm{Hм}$ (рис. 2-7,a) и 840 нм (рис. 7,b-9). В структуре, излучающей на 808 нм, материалами КЯ и волноводной области являются (GaAs) $)_{0.9}\left(\mathrm{Ga}_{0.51} \mathrm{In}_{0.49} \mathrm{P}\right)_{0.1}$ и $(\mathrm{GaAs})_{0.46}\left(\mathrm{Ga}_{0.51} \mathrm{In}_{0.49} \mathrm{P}\right)_{0.54}$ соответственно; ширина КЯ равна 7.4 нм. В структуре, излучающей на 840 нм, материалами КЯ и волноводной области являются GaAs и $\mathrm{Al}_{0.2} \mathrm{Ga}_{0.8} \mathrm{As}$ соответственно [3,9]; ширина КЯ равна 7.5 нм. Ширина волноводной области в обеих структурах $b=0.4$ мкм. Длина резонатора $L=500$ мкм и ширина полоскового контакта $W=3$ мкм.

В предположении локальной электронейтральности в КЯ $[3,9]$ концентрация электронов и дырок в КЯ оставалась неизменной с ростом тока инжекции и равной пороговому значению. Как видно из уравнения (24), учет нарушения локальной электронейтральности в КЯ приводит к зависимости концентрации электронов в КЯ, а следовательно и концентрации дырок (см. формулу $(20))$, от плотности тока инжекции $j$. Как видно из условия лазерной генерации $(19), n^{\mathrm{QW}}$ и $p^{\mathrm{QW}}$ не могут одновременно возрастать или убывать. Если $n$ QW уменьшается с ростом тока, то выполнение условия генерации (19) требует увеличения $p^{\mathrm{QW}}$ и наоборот.

На рис. 2 показаны $n^{\mathrm{QW}}$ и $p^{\mathrm{QW}}$ в зависимости от плотности тока инжекции. Как видно из рисунка, в рассматриваемой структуре $n^{\mathrm{QW}}$ уменьшается, а $p^{\mathrm{QW}}$ увеличивается с ростом тока. Таким образом, разность между $n^{\mathrm{QW}}$ и $p^{\mathrm{QW}}$ возрастает (т.е. нарушение локальной электронейтральности проявляется сильнее) с увеличением тока накачки. Тем не менее из рисунка видно, 


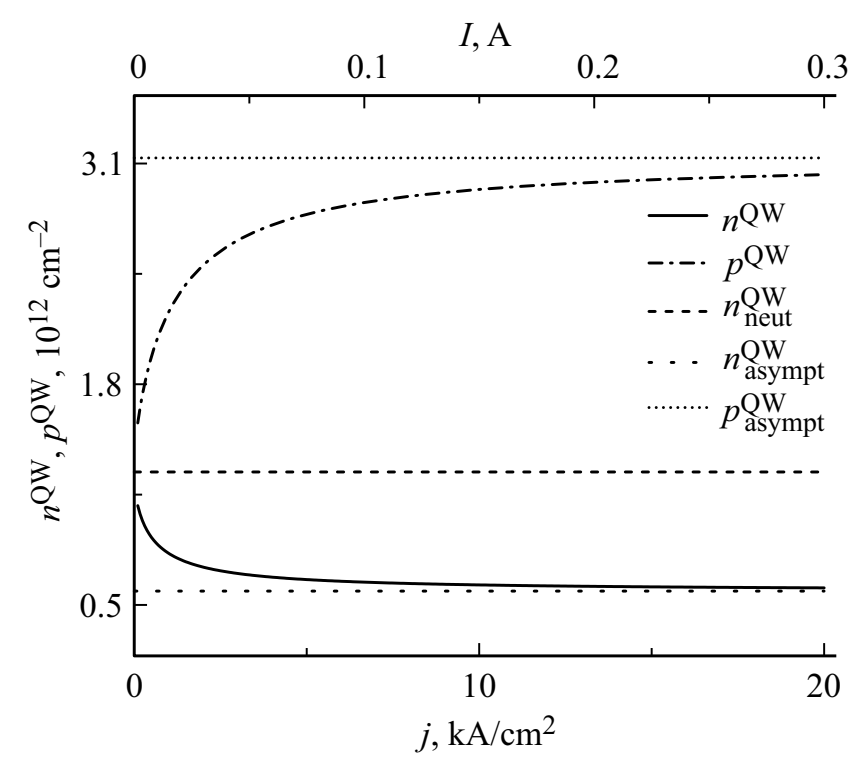

Рис. 2. Двумерные концентрации электронов (сплошная кривая) и дырок (штрихпунктирная кривая) в КЯ в зависимости от плотности тока инжекции (нижняя ось) и тока инжекции (верхняя ось). Горизонтальная штриховая прямая изображает концентрацию электронов и дырок в КЯ, рассчитанную в предположении локальной электронейтральности в КЯ. Горизонтальные пунктирные прямые изображают асимптотические значения электронной и дырочной концентраций в КЯ, даваемые формулами (38) и (40). На рис. 2-7, $a$ приведены результаты расчетов для структуры, излучающей на длине волны $\lambda_{0}=808$ нм. На рис. 2-9 ширина волноводной области $b=0.4$ мкм, длина резонатора $L=500$ мкм, ширина полоскового контакта $W=3$ мкм.

что как падение $n^{\mathrm{QW}}$, так и рост $p^{\mathrm{QW}}$ насыщаются с увеличением $j: n^{\mathrm{QW}}$ и $p^{\mathrm{QW}}$ приближаются к своим асимптотическим значениям (эти значения показаны пунктирными горизонтальными прямыми на рисунке). Из (24) легко получить следующее выражение для асимптотического значения $n^{\mathrm{QW}}$ при $j \rightarrow \infty$ :

$$
n_{\mathrm{asympt}}^{\mathrm{QW}}=N_{c}^{2 \mathrm{D}} \ln \left(\frac{1+\frac{\tau_{p, \text { capt }, 0}}{\tau_{n, \text { capt }, 0}}}{1-\frac{\beta}{g^{\max }}}\right),
$$

где

$$
\tau_{n, \text { capt }, 0}=\frac{b_{1}}{v_{n, \text { capt }, 0}}, \quad \tau_{p, \text { capt }, 0}=\frac{b_{2}}{v_{p, \text { capt }, 0}}
$$

являются временами захвата в незаполненную КЯ электронов и дырок из левой и правой частей волноводной области соответственно.

Используя (38) в (20), для асимптотического значения $p^{\mathrm{QW}}$ при $j \rightarrow \infty$ имеем

$$
p_{\mathrm{asympt}}^{\mathrm{QW}}=N_{v}^{2 \mathrm{D}} \ln \left(\frac{1+\frac{\tau_{n, \text { capt }, 0}}{\tau_{p, \text { capt }, 0}}}{1-\frac{\beta}{g^{\max }}}\right) .
$$

Мы используем в этой статье одинаковые значения скоростей захвата электронов и дырок в КЯ $\left(v_{n, \text { capt }, 0}=v_{p, \text { capt }, 0}=1 \cdot 10^{5} \mathrm{~cm} / \mathrm{c}\right)$, а также полагаем, что КЯ помещена в центре волноводной области, т.е. используем одинаковые значения толщин левой и правой частей волноводной области $\left(b_{1}=b_{2}=b / 2=0.2\right.$ мкм). Таким образом, времена захвата электронов и дырок в КЯ одинаковы $\left(\tau_{n, \text { capt }, 0}=\tau_{p \text {, capt }, 0}=0.2 \mathrm{нc}\right)$. Как видно из (38) и (40), в случае равных $\tau_{n \text {, сарt,0 }}$ и $\tau_{p, \text { capt, } 0}$ максимальное отношение положительного заряда в КЯ к отрицательному равно

$$
\frac{e p_{\mathrm{asympt}}^{\mathrm{QW}}}{e n_{\mathrm{asympt}}^{\mathrm{QW}}}=\frac{N_{v}^{2 \mathrm{D}}}{N_{c}^{2 \mathrm{D}}}=\frac{m_{\mathrm{hh}}^{\mathrm{QW}}}{m_{c}^{\mathrm{QW}}},
$$

где мы использовали выражения для $N_{c, v}^{2 \mathrm{D}}$, приведенные после формул (6). Для рассмотренной здесь КЯ на основе $(\mathrm{GaAs})_{0.9}\left(\mathrm{Ga}_{0.51} \mathrm{In}_{0.49} \mathrm{P}\right)_{0.1} p_{\text {asympt }}^{\mathrm{QW}} / n_{\text {asympt }}^{\mathrm{QW}}=5.4$ (см. пунктирные горизонтальные прямые на рис. 2). Таким образом, наши расчеты показывают, что локальная электронейтральность в КЯ существенным образом нарушена, особенно при высоких токах накачки.

На рис. 3 показаны степени заполнения состояний, соответствующих нижнему краю электронной и верхнему краю дырочной подзон размерного квантования в КЯ, в зависимости от плотности тока накачки. Характер этих зависимостей очевиден из формул (6) и рис. $2-$ поскольку $n^{\mathrm{QW}}$ убывает, то убывает и $f_{n}$, поскольку $p^{\mathrm{QW}}$ растет, то растет и $f_{p}$. С ростом тока $f_{n}$ и $f_{p}$ прибли-

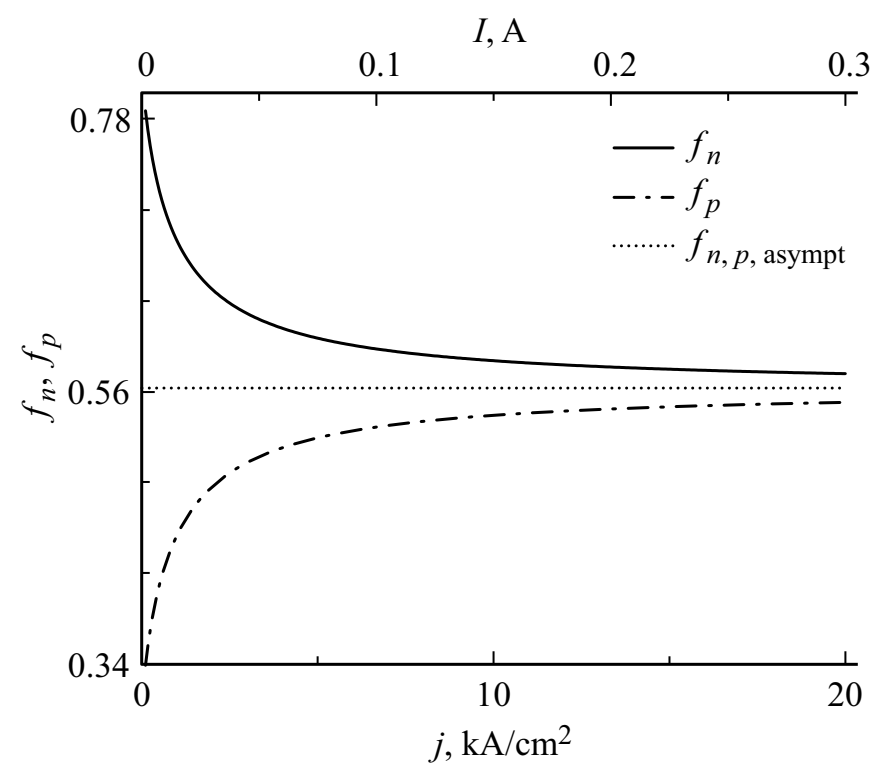

Рис. 3. Степени заполнения состояний, соответствующих нижнему краю электронной (сплошная кривая) и верхнему краю дырочной (штрихпунктирная кривая) подзон размерного квантования в КЯ в зависимости от плотности тока инжекции (нижняя ось) и тока инжекции (верхняя ось). Горизонтальная точечная прямая изображает асимптотические значения степеней заполнения, даваемые формулами (42), (43) и совпадающие друг с другом при совпадении времен захвата электронов и дырок в яму. 
жаются к следующим асимптотическим значениям:

$$
\begin{aligned}
& f_{n, \text { asympt }}=\frac{\frac{\beta}{g^{\max }}+\frac{\tau_{p, \text { capt }, 0}}{\tau_{n, \text { capt }, 0}}}{1+\frac{\tau_{p, \text { capt }, 0}}{\tau_{n, \text { capt }, 0}}}, \\
& f_{p, \text { asympt }}=\frac{\frac{\beta}{g^{\max }}+\frac{\tau_{n, \text { capt }, 0}}{\tau_{p, \text { capt }, 0}}}{1+\frac{\tau_{n, \text { capt }, 0}}{\tau_{p, \text { capt }, 0}}} .
\end{aligned}
$$

В случае равных $\tau_{n \text {,capt, } 0}$ и $\tau_{p \text {,capt, } 0}$ асимптотические значения, даваемые формулами (42) и (43), совпадают (см. пунктирную горизонтальную прямую на рис. 3).

Ввиду превышения заряда дырок в КЯ над зарядом электронов (рис. 2) условие глобальной электронейтральности (12) требует превышения заряда $e b_{1} n_{\mathrm{L}}^{\mathrm{OCL}}$ электронов в левой части волноводной области над зарядом $e b_{2} p_{\mathrm{R}}^{\mathrm{OCL}}$ дырок в ее правой части. На рис. 4 показаны концентрации свободных электронов и дырок в левой и правой частях волноводной области соответственно в зависимости от плотности тока инжекции. Для сравнения с двумерными концентрациями носителей в КЯ (рис. 2) концентрации свободных носителей пересчитаны на единицу площади контакта, т.е. на рис. 4 приведены величины $b_{1} n_{\mathrm{L}}^{\mathrm{OCL}}$ и $b_{2} p_{\mathrm{R}}^{\mathrm{OCL}}$. Как видно из pис. 2 и 4 , величины $b_{1} n_{\mathrm{L}}^{\mathrm{OCL}}$ и $b_{2} p_{\mathrm{R}}^{\mathrm{OCL}}$ близки друг к другу и каждая из них существенно выше концентраций электронов и дырок в КЯ $n^{\mathrm{QW}}$ и $p^{\mathrm{QW}}$. Таким образом, небольшое относительное превышение $n_{\mathrm{L}}^{\mathrm{OCL}}$ над $p_{\mathrm{R}}^{\mathrm{OCL}}$ (рис. 4) компенсирует существенное превышение $p^{\mathrm{QW}}$ над $n^{\mathrm{QW}}$ (рис. 2).

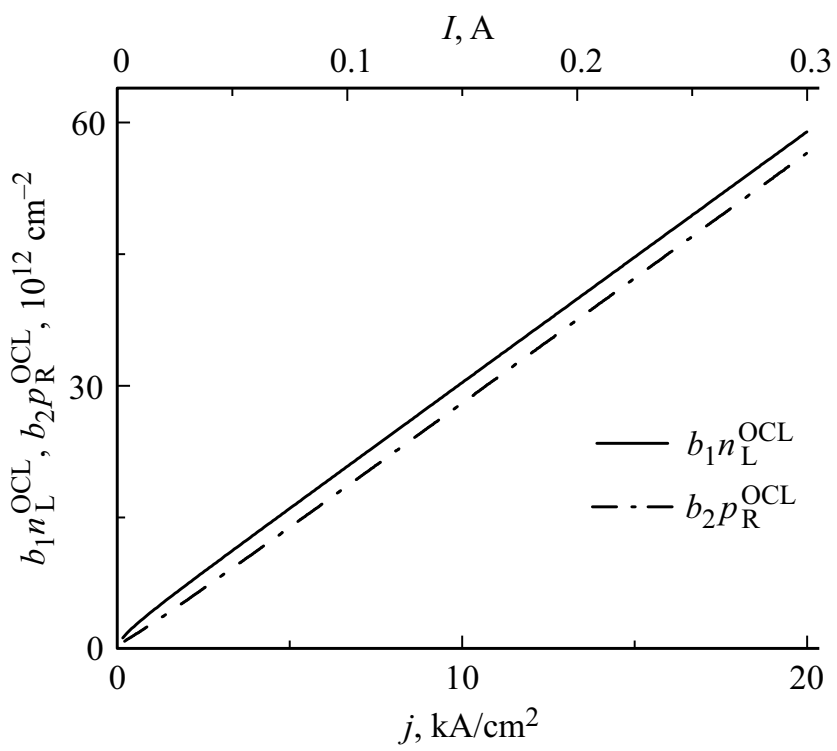

Рис. 4. Концентрации свободных электронов в левой части волноводной области (сплошная кривая) и дырок в правой ее части (штрихпунктирная кривая) на единицу площади контакта в зависимости от плотности тока инжекции (нижняя ось) и тока инжекции (верхняя ось).

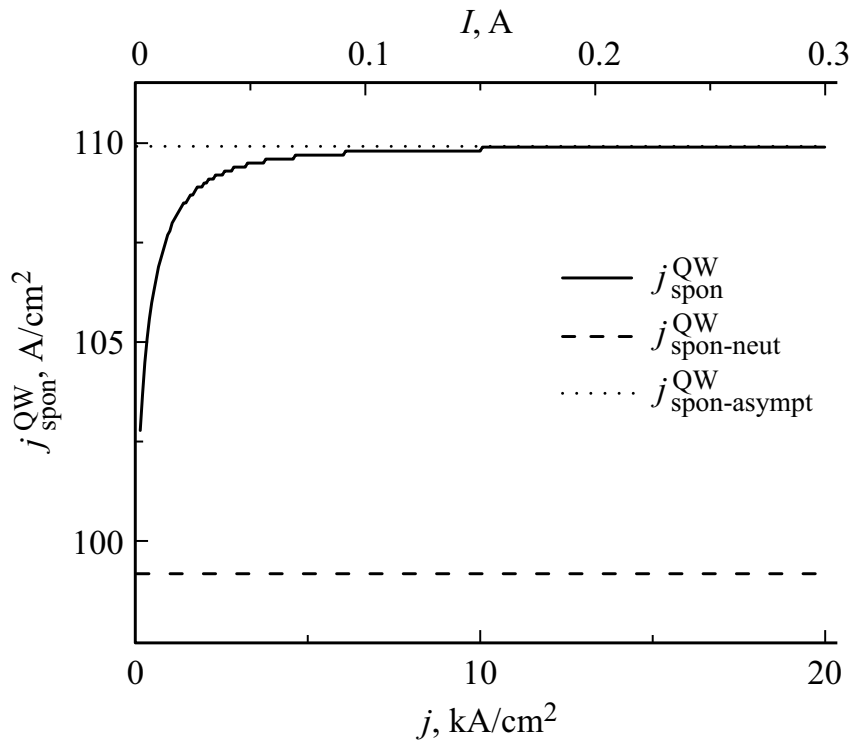

Рис. 5. Плотность тока спонтанной излучательной рекомбинации в КЯ в зависимости от плотности тока инжекции (нижняя ось) и тока инжекции (верхняя ось). Горизонтальная штриховая прямая изображает плотность тока спонтанной излучательной рекомбинации в КЯ, рассчитанную в предположении локальной электронейтральности в КЯ. Горизонтальная пунктирная прямая изображает асимптотическое значение плотности тока спонтанной излучательной рекомбинации в КЯ, даваемое формулой (45).

Асимптотическое выражение для $b_{1} n_{\mathrm{L}}^{\mathrm{OCL}}$ и $b_{2} p_{\mathrm{R}}^{\mathrm{OCL}}$ при $j \rightarrow \infty$ имеет следующий вид:

$$
b_{1} n_{\mathrm{L}, \text { asympt }}^{\mathrm{OCL}}=b_{2} p_{\mathrm{R}, \text { asympt }}^{\mathrm{OCL}}=\frac{\tau_{n, \mathrm{capt}, 0}+\tau_{p, \text { capt }, 0}}{1-\frac{\beta}{g^{\max }}} \frac{j}{e} .
$$

Ввиду того что концентрации электронов и дырок в КЯ зависят от плотности тока инжекции, плотность тока спонтанной излучательной рекомбинации в КЯ также зависит от $j$ (рис. 5): как видно из рис. $5, j$ spon растет с ростом $j$. Однако, в отличие от существенных вариаций $n^{\mathrm{QW}}$ и $p^{\mathrm{QW}}$ с током (рис. 2 ), рост $j_{\text {spon }}^{\mathrm{QW}}$ является незначительным: при изменении $j$ от $j$ th $\left(103 \mathrm{~A} / \mathrm{cm}^{2}\right)$ до $20 \mathrm{\kappa A} / \mathrm{cm}^{2} j_{\text {spon }}^{\mathrm{QW}}$ меняется от 103 до $110 \mathrm{~A} / \mathrm{cm}^{2}$. Как видно из выражения (35), такое поведение $j$ spon является следствием того, что рост $p^{\mathrm{QW}}$ с током в значительной степени компенсируется падением $n^{\mathrm{QW}}$ (рис. 2). Так как $n^{\mathrm{QW}}$ и $p^{\mathrm{QW}}$ насыщаются с ростом тока, $j_{\text {spon }}^{\mathrm{QW}}$ также насыщается. Используя (38) и (40), для асимптотического значения $j_{\text {spon }}^{\mathrm{QW}}$ при $j \rightarrow \infty$ имеем (пунктирная горизонтальная прямая на рис. 5)

$$
j_{\text {spon, asympt }}^{\mathrm{QW}}=e B_{2 \mathrm{D}} N_{c}^{2 \mathrm{D}} N_{v}^{2 \mathrm{D}} \ln \left(\frac{1+\frac{\tau_{p, \text { capt }, 0}}{\tau_{n, \text { capt }, 0}}}{1-\frac{\beta}{g^{\max }}}\right) \ln \left(\frac{1+\frac{\tau_{n, \text { capt }, 0}}{\tau_{p, \text { capt }, 0}}}{1-\frac{\beta}{g^{\max }}}\right) .
$$




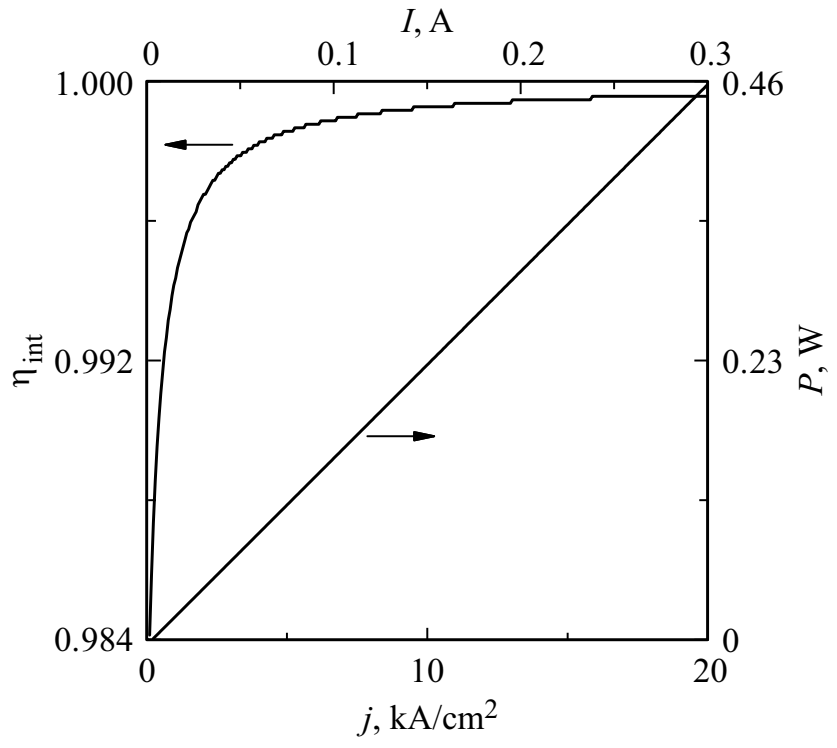

Рис. 6. Внутренняя дифференциальная квантовая эффективность (левая ось) и выходная оптическая мощность (правая ось) в зависимости от плотности тока инжекции (нижняя ось) и тока инжекции (верхняя ось).

Поскольку плотность тока спонтанной излучательной рекомбинации в КЯ низка и остается ограниченной с увеличением тока накачки (рис. 5), то, как видно из (34), внутренняя дифференциальная квантовая эффективность должна приближаться к единице, т.е. возрастать с ростом тока накачки, что и показано на рис. 6 (левая ось). Как видно из рисунка, $\eta_{\text {int }}$ близка к единице уже на пороге генерации, так что ее возрастание является незначительным.

На рис. 6 также показана мощность выходного оптического излучения лазера (правая ось) в зависимости от плотности тока накачки. Как видно из рисунка, зависимость является линейной и неотличимой от ВТАХ, рассчитанной в предположении локальной электронейтральности в КЯ [9]. Близость $\eta_{\text {int }}$ к единице (рис. 6) и линейность ВтАХ (как и в случае локальной нейтральности в КЯ) являются следствием предположения об идеальном функционировании АБС, когда рекомбинации в волноводной области полностью подавлена.

На рис. 7 показана характеристическая температура лазера, определяемая как [11,22]

$$
T_{0}=\left(\frac{\partial \ln j_{\text {th }}}{\partial T}\right)^{-1},
$$

в зависимости от рабочей температуры для структур, излучающих на 808 и 840 нм. Для сравнения на рисунке также показана (штриховая прямая) $T_{0}$, рассчитанная в предположении зарядовой нейтральности в КЯ [3]. Как было показано в работе [3], в случае полного подавления рекомбинации вне активной области (случай, рассматриваемый и в данной статье)

$$
T_{0, \text { neut }}=T \text {. }
$$

Как видно из рисунка, $T_{0}$, рассчитанная с учетом нарушения зарядовой нейтральности в КЯ, выше, чем $T_{0 \text {,neut, }}$

$$
T_{0}>T_{0, \text { neut }}
$$

Превышение $T_{0}$ над $T_{0 \text {, neut }}$ существеннее в структуре, излучающей на 840 нм (рис. 7, $b$ ).

Этот результат, означающий, что нарушение зарядовой нейтральности в КЯ приводит к ослаблению температурной зависимости порогового тока (что показано на рис. 8), не является самоочевидным. Как видно из (36), для того чтобы это имело место, необходимо, чтобы произведение $n_{\mathrm{th}}^{\mathrm{QW}} p_{\mathrm{th}}^{\mathrm{QW}}$ росло с ростом температуры медленнее, чем $\left(n_{\mathrm{th} \text {, neut }}^{\mathrm{QW}}\right)^{2}$, где $n_{\mathrm{th} \text {, neut }}^{\mathrm{QW}}-$ концентрация электронов и дырок в случае зарядовой нейтральности в КЯ. На рис. 9 показаны $n_{\mathrm{th}}^{\mathrm{QW}}, p_{\mathrm{th}}^{\mathrm{QW}}$ и $n_{\text {th, neut }}^{\mathrm{QW}}$ в зависимости от температуры. Из рисунка видно, что в то время как концентрация электронов $n_{\mathrm{th}}^{\mathrm{QW}}$ растет с ростом темпе-
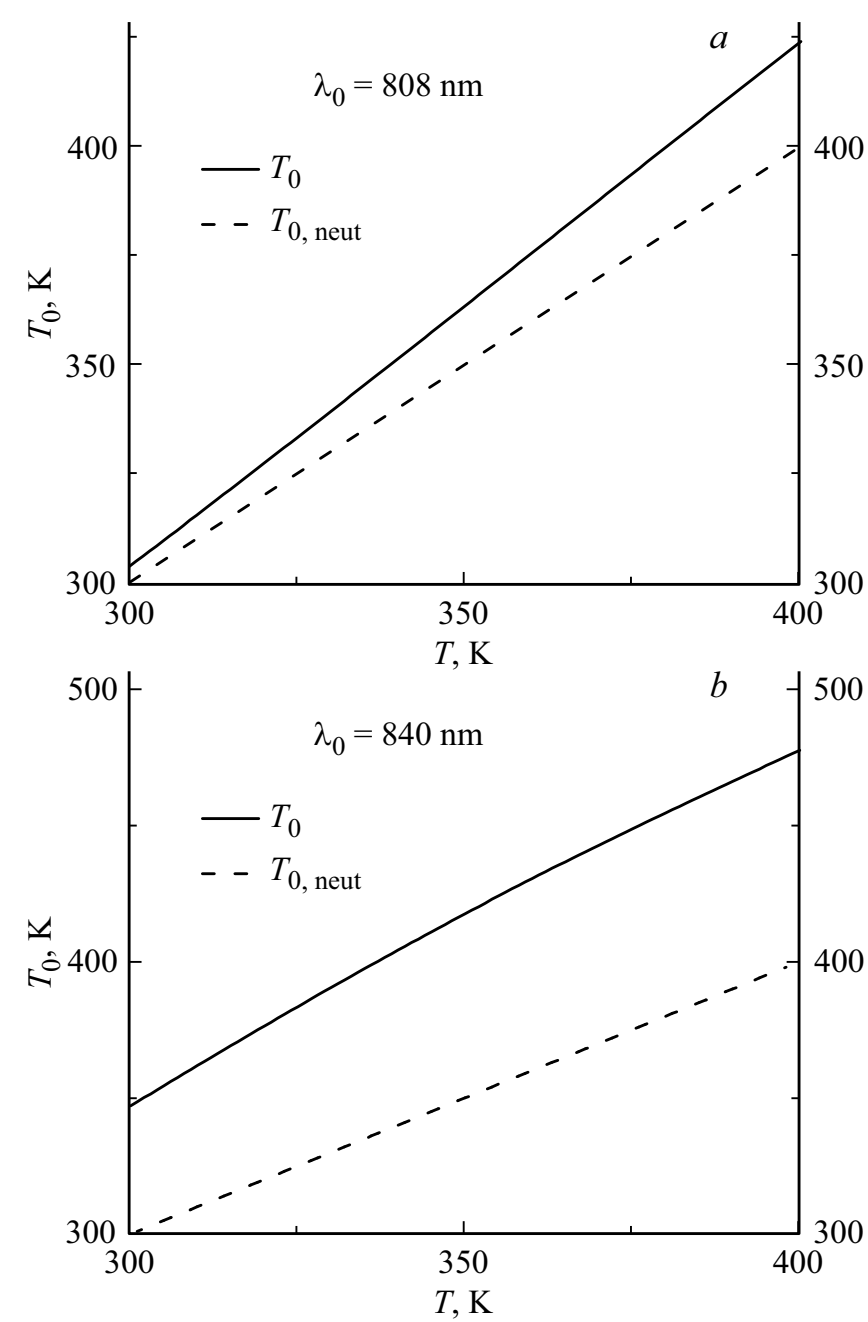

Рис. 7. Характеристическая температура лазера с асимметричными барьерными слоями в зависимости от рабочей температуры для структур, излучающих на 808 (a) и 840 нм (b). Штриховая прямая изображает характеристическую температуру, рассчитанную в предположении локальной электронейтральности в КЯ. 


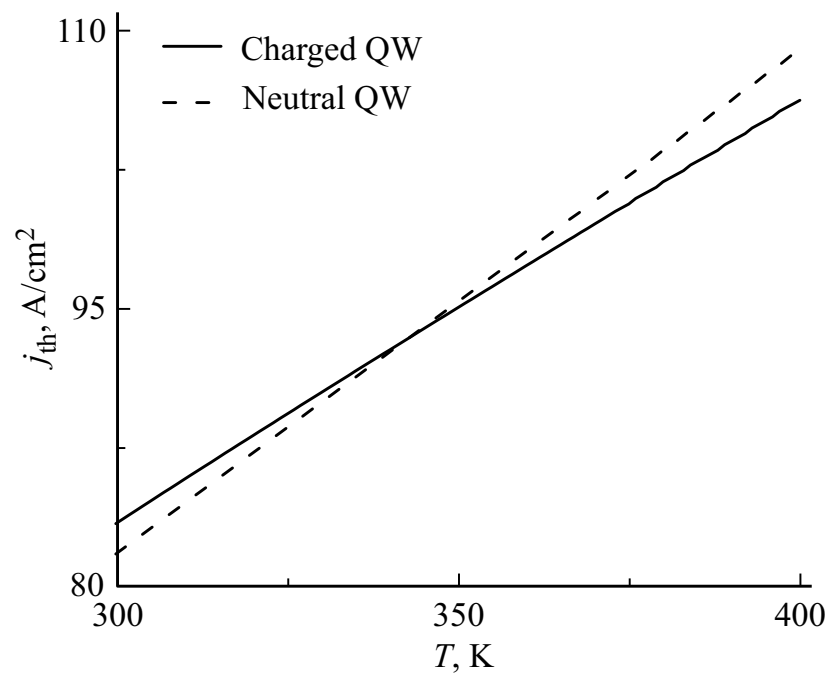

Рис. 8. Плотность порогового тока лазера с асимметричными барьерными слоями в зависимости от температуры. Штриховая прямая изображает плотность порогового тока, рассчитанную в предположении локальной электронейтральности в КЯ. На рис. 8 и 9 приведены результаты расчетов для структуры, излучающей на длине волны $\lambda_{0}=840$ нм.

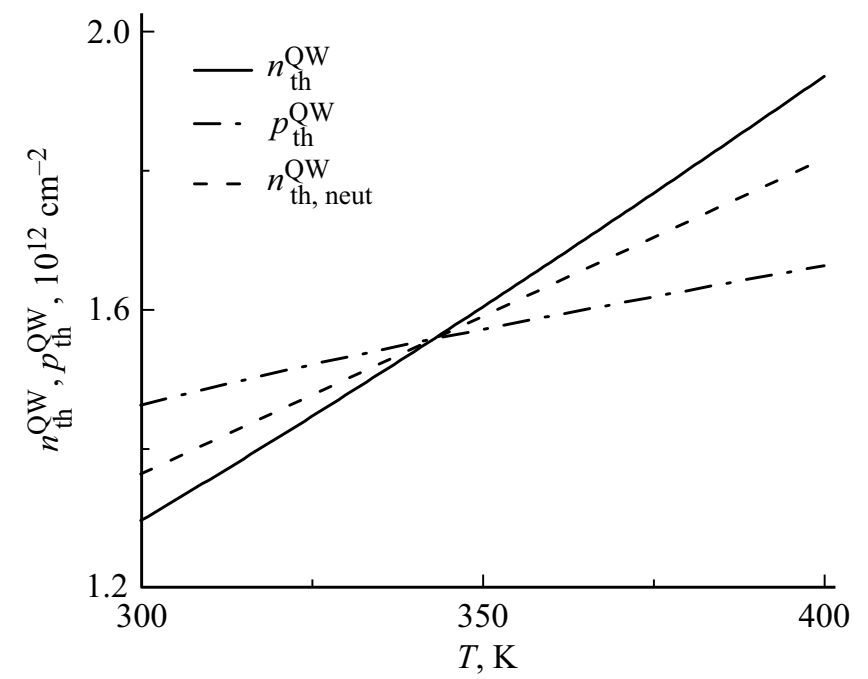

Рис. 9. Пороговые концентрации электронов (сплошная кривая) и дырок (штрихпунктирная кривая) в КЯ в зависимости от температуры. Штриховая прямая изображает пороговую концентрацию электронов и дырок, рассчитанную в предположении локальной электронейтральности в КЯ.

ратуры быстрее, чем $n_{\mathrm{th}, \text { neut }}^{\mathrm{QW}}$, концентрация дырок $p_{\mathrm{th}}^{\mathrm{QW}}$ возрастает существенно медленнее, чем $n_{\mathrm{th}, \text { neut }}^{\mathrm{QW}}$, образом, $n_{\mathrm{th}}^{\mathrm{QW}} p_{\mathrm{th}}^{\mathrm{QW}}$ увеличивается с ростом температуры действительно медленнее, чем $\left(n_{\text {th, neut }}^{\mathrm{QW}}\right)^{2}$.

\section{4. Заключение}

Разработана самосогласованная теоретическая модель для расчета пороговых и мощностных характеристик полупроводниковых лазеров на КЯ с АБС. Модель, основанная на системе скоростных уравнений, использует универсальное условие глобальной зарядовой нейтральности в лазерной структуре. Рассчитаны концентрации электронов и дырок в волноводной области и в КЯ и ватт-амперная характеристика лазера. Показано, что локальная нейтральность в КЯ сильно нарушена, особенно при высоких токах инжекции. Нарушение нейтральности в КЯ приводит к зависимости концентраций электронов и дырок в ней от тока инжекции в режиме лазерной генерации - в рассмотренной нами структуре концентрация электронов в КЯ уменьшается, a концентрация дырок увеличивается с ростом тока инжекции. В условиях идеального функционирования асимметричных барьерных слоев, когда имеет место полное подавление электронно-дырочной рекомбинации в волноводной области, нарушение нейтральности в КЯ практически не сказывается на внутренней дифференциальной квантовой эффективности и ВтАХ лазера квантовая эффективность близка к единице, а ВтАХ линейна. Нарушение нейтральности в КЯ приводит тем не менее к ослаблению температурной зависимости порогового тока и, таким образом, повышению характеристической температуры $T_{0}$ лазера.

Разработанная нами теоретическая модель может служить для дальнейшей оптимизации лазерных структур с АБС.

Работа выполнена при поддержке Российского научного фонда (проект 14-42-00006 „Новый тип полупроводниковых лазеров с характеристиками, улучшенными за счет использования асимметричных барьеров“"). Л.В. Асрян также благодарит Исследовательский офис армии США (U.S. Army Research Office, грант № W911NF-17-1-0432).

\section{Список литературы}

[1] L.V. Asryan, S. Luryi. Solid-State Electron., 47 (2), 205 (2003).

[2] L.V. Asryan, S. Luryi. U.S. Patent N 6, 870, 178, Mar. 22, 2005; (U.S. Provisioal Patent Application N 60/272, 202, filed on Feb. 28, 2001).

[3] L.V. Asryan, N.V. Kryzhanovskaya, M.V. Maximov, A.Yu. Egorov, A.E. Zhukov. Semicond. Sci. Technol., 26 (5), 055025 (2011).

[4] A.E. Zhukov, N.V. Kryzhanovskaya, F.I. Zubov, Y.M. Shernyakov, M.V. Maximov, E.S. Semenova, K. Yvind, L.V. Asryan. Appl. Phys. Lett., 100 (2), 021107 (2012).

[5] L.V. Asryan, S. Luryi. IEEE J. Quant. Electron., 37 (7), 905 (2001).

[6] D.-S. Han, L.V. Asryan. Appl. Phys. Lett., 92 (25), 251113 (2008).

[7] D.-S. Han, L.V. Asryan. J. Lightw. Technol., 27 (24), 5775 (2009).

[8] D.-S. Han, L.V. Asryan. Nanotechnology, 21 (1), 015201 (2010).

[9] Л.В. Асрян, Ф.И. Зубов, Н.В. Крыжановская, М.В. Максимов, А.Е. Жуков. ФТП, 50 (10), 1380 (2016).

[10] L.V. Asryan, R.A. Suris. IEEE J. Select. Topics Quant. Electron., 3 (2), 148 (1997). 
[11] L.V. Asryan, R.A. Suris. Electron. Lett., 33 (22), 1871 (1997).

[12] З.Н. Соколова, Н.А. Пихтин, И.С. Тарасов, Л.В. Асрян. Квант. электрон., 46 (9), 777 (2016).

[13] З.Н. Соколова, Д.А. Веселов, Н.А. Пихтин, И.С. Тарасов, Л.В. Асрян. ФТП, 51 (7), 998 (2017).

[14] K.J. Vahala, C.E. Zah. Appl. Phys. Lett., 52 (23), 1945 (1988).

[15] L.V. Asryan, S. Luryi. Appl. Phys. Lett., 83 (26), 5368 (2003).

[16] L.V. Asryan, S. Luryi. IEEE J. Quant. Electron., 40 (7), 833 (2004).

[17] Л.В. Асрян. Квант. электрон., 35 (12), 1117 (2005).

[18] L.V. Asryan, Z.N. Sokolova. J. Appl. Phys., $115(2), 023107$ (2014).

[19] L.V. Asryan, S. Luryi, R.A. Suris. Appl. Phys. Lett., 81 (12), 2154 (2002).

[20] L.V. Asryan, S. Luryi, R.A. Suris. IEEE J. Quant. Electron., 39 (3), 404 (2003)

[21] L.A. Coldren, S.W. Corzine. Diode Lasers and Photonic Integrated Circuits (N.Y., Wiley, 1995).

[22] L.V. Asryan, R.A. Suris. IEEE J. Quant. Electron., 34 (5), 841 (1998).

Редактор А.Н. Смирнов

\section{Violation of local electroneutrality in the quantum well of a semiconductor laser with asymmetric barrier layers}

L.V. Asryan ${ }^{1}$, F.I. Zubov' Yu.S. Balezina (Polubavkina) ${ }^{2}$, E.I. Moiseev², M.E. Muretova ${ }^{2}$, N.V. Kryzhanovskaya ${ }^{2}$, M.V. Maximov' ${ }^{2}$, A.E. Zhukov ${ }^{2}$

${ }^{1}$ Virginia Polytechnic Institute and State University, Blacksburg, Virginia 24061, USA

${ }^{2}$ St. Petersburg National Research Academic University, Russian Academy of Sciences, 194021 St. Petersburg, Russia

Abstract A self-consistent model for calculation of threshold and power characteristics of semiconductor quantum well $(\mathrm{QW})$ lasers with asymmetric barrier layers is developed. The model, which is based on a set of rate equations, uses the universal condition of global charge neutrality in the laser structure. The electron and hole densities in the waveguide region and in the QW and density of photons of stimulated emission are calculated. The local neutrality in the QW is shown to be strongly violated, especially at high injection currents. Violation of neutrality in the QW makes the electron and hole densities in the QW dependent on the injection current in the lasing regime - in the structure considered here, the electron density in the QW decreases while the hole density increases with increasing injection current. Under the conditions of ideal functioning of the asymmetric barrier layers, when the electron-hole recombination in the waveguide region is totally suppressed, violation of neutrality in the QW does not practically affect the dependence of the output optical power on the injection current - the quantum efficiency is close to 1 and the light-current characteristic is linear. Violation of neutrality in the QW weakens, however, the temperature-dependence of threshold current and hence makes the characteristic temperature $T_{0}$ of the laser higher. 